\title{
Dwarf Galaxies and the Cosmic chemical Matter Cycle
}

\section{Gerhard Hensler*}

University of Vienna, Dept. of Astrophysics, Tuerkenschanzst. 17, 1180 Vienna, Austria

E-mail: gerhard.henslereunivie.ac.at

\section{Simone Recchi}

University of Vienna, Dept. of Astrophysics

E-mail: simone.recchi@univie.ac.at

To understand the lower element abundances of Dwarf Galaxies (DGs) according to a massmetallicity relation, gas loss through galactic winds is commonly invoked. For simplification, the galactic mass arises to become the principal parameter affecting the chemical evolution of DGs. In contrast, mixing effects between the gas phases, the gas distribution and its state strongly influence the mass loss efficiency. In addition, environmental processes as e.g. gas infall and external tides also contribute to the chemical evolution. In particular, at low gravitational energies as in DGs energetic effects dominate the evolution and enhance the chemical and structural signatures. Dynamics and gas-phase mixing processes determine the galactic chemical evolution and must, therefore, be treated properly, and studied in detail.

By means of a 2D and recently developed 3D chemo-dynamical treatment of galaxy evolution we explore the influence of different internal and environmental processes on the structure and element abundances from numerical models of DGs. Here we can present only two results of our multiple diverse studies, which demonstrate that detailed explorations of the various processes and their intimate coupling with chemical elements are necessary to account for the evolutionary history of galaxies.

XII International Symposium on Nuclei in the Cosmos,

August 5-12, 2012

Cairns, Australia

${ }^{*}$ Speaker. 


\section{Introduction}

The steepening slope of the mass-metallicity relation in the dwarf galaxies (DGs) regime [13] is interpreted by galactic mass loss via winds [4] and the corresponding lower effective yield $y_{\text {eff }}$. Nevertheless, the full truth of their evolution, namely, hot-gas outflows vs. infall of primordial gas and both including gas mixing and heating vs. cooling effects, is concealed in the abundance ratios of particular elements released by stars on various timescales and via different stellar processes. How environmental gasdynamical effects influence the chemical evolution of DGs is shown here for two evolutionary scenarios, one semi-analytically for low-metallicity gas infall igniting star formation (SF) and affecting abundance ratios, and secondly, exploiting the influence of the gas distribution on the development of galactic winds.

\section{Gas infall}

DGs show present-day abundance ratios, as e.g. O/Fe and N/O, that are discrepant from the solar vicinity and have also to be understood. In particular, DGs in the Milky Way neighbourhood reach already solar values of $[\mathrm{O} / \mathrm{Fe}]$ for subsolar $\mathrm{O}$ or $\mathrm{Fe}$ abundances though starting from the same ratio typical for their roots in massive stars. The behaviour, that $[\mathrm{O} / \mathrm{Fe}]$ already leaves the flat relation stemming from short-living massive stars much below $[\mathrm{Fe} / \mathrm{H}] \approx-1.0$ (see e.g. [12]) and drops to solar can be explained by a long SF timescale and loss of metal-enriched gas as well as by infall of unprocessed gas.

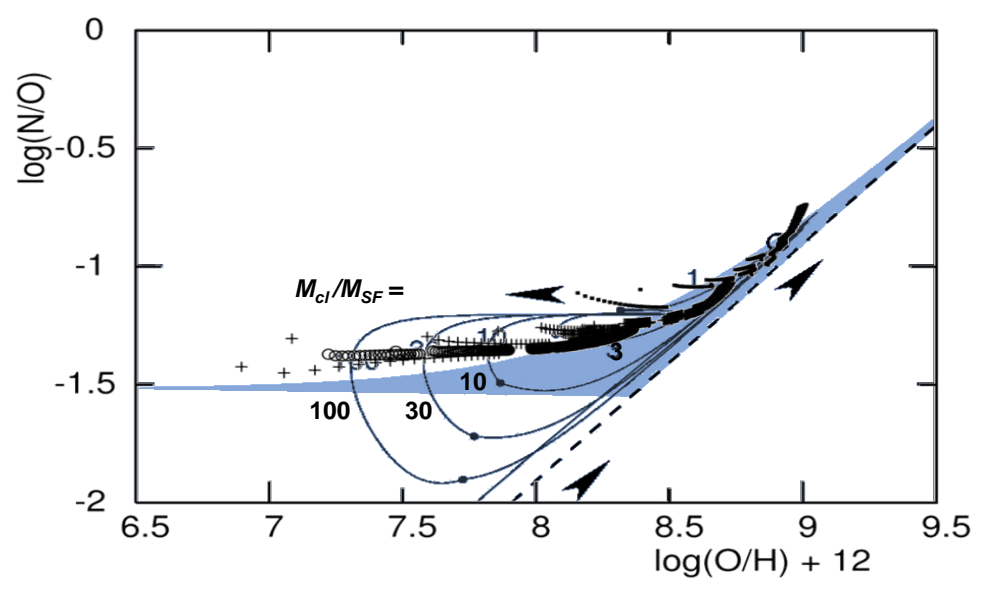

Figure 1: The abundance ratio $\mathrm{N} / \mathrm{O}$ as a function of oxygen abundance observed in spiral and irregular galaxies (shaded area, after [14]) overlayed with evolutionary loops due to infall of primordial intergalactic gas clouds. The loop size depends on the mass ratio of the cloud mass $M_{c l}$ and the local gas mass $M_{S F}$ involved in the SF region. The crosses represent evolutionary timesteps of models and the arrows depict the direction of the evolutionary paths. The dashed straight line represents a simple model relation for purely secondary nitrogen production. For a detailed discussion see [3].

The ratio $\log (\mathrm{N} / \mathrm{O})$ stays for DGs at about -1.6 to -1.5 with $\mathrm{O}$ abundances below $1 / 10$ solar and with an increasing scatter with $\mathrm{O}$ (see Fig.1). Their regime of N/O-O/H values overlaps with 
those of HII regions in the outermost disk parts of spirals at about $12+\log (\mathrm{O} / \mathrm{H})=8.0 \quad \ldots 8.5$ [14]. Although this can be understood for the abundance enrichment by young stellar populations as for damped Lyman $\alpha$ absorbers by yields of rotating massive stars [1], these DGs contain also older stellar population, so that a much less efficient SF with respect to solar environmental conditions or another process must be favoured. Since these objects are embedded into HI envelops and are suggested to suffer gas infall as e.g. NGC 1569 [5], the influence of metal-poor gas infall into an old galaxy with continuous SF on particular abundance patterns were exploited by [3].

That primary production dominates in the early phases of chemical evolution and secondary production in more evolved stages appears to be somewhat difficult to reconcile with what is known from stellar nucleosynthesis studies. While secondary nitrogen production occurs in massive stars, the predominant contribution of primary production comes from intermediate-mass stars due to the Third Dredge-Up mixing freshly synthesised carbon into higher layers, where it can be turned by hot-bottom burning to nitrogen. Nevertheless, a not insignificant primary $\mathrm{N}$ production occurs in short-living massive stars and also included in this analtical model. Thus, once the system is evolved enough for all intermediate-mass stars to contribute and the SF continues, the N/O ratio would remain rather constant instead of increasing with O/H. Fig.1 demonstrates how self-enriched galaxies which have reached the secondary N-track already within 2-3 Gyrs of their evolution are thrown back in $\mathrm{O}$ abundance by gas infall while N/O stays the same. After a time delay depending on the mass fraction of infalling gas to that existing already within the SF site, along a loop-like evolutionary paths in the $[12+\log (\mathrm{O} / \mathrm{H})]-\log (\mathrm{N} / \mathrm{O})$ diagram the ISM abundances reach again the starting point. With the reasonable assumption that the fraction of infalling-to-existing gas mass increases with decreasing galaxy mass, their results could match not only the observational regime of BCDs in the $[12+\log (\mathrm{O} / \mathrm{H})]-\log (\mathrm{N} / \mathrm{O})$ space but also explain the shark-fin shape of observational data distribution [14]. This infall process provides an alternative scenario to other models in which old dIrrs mutate temporarily to youthfully appearing examples due to their rejuvenated gas abundances.

\section{Galactic winds in different gas shapes}

Not only that large-scale outflows of hot gas in DGs reduce their element abundances by mixing effects with cold gas [6] and are mass-loaded through the evaporation of embedded clouds [7], their development considerably depends on the gas distribution, i.e. on the degree of flattening associated with rotation. Disk-like galaxies tend to develop outflows perpendicularly to the disk. Preferential outflow directions do not exist for spherical galaxies, which thus either eject gas isotropically (but complete blow-away is almost impossible in DGs [2]), or do not eject gas at all. Surprisingly, the dependence of ejection efficiencies (of gas and freshly produced metals) on the gas distribution has received little attention in the past (but see [11], [9], [10]).

We have carried out a series of 2-D high-resolution hydrodynamical simulations of DGs to fill this gap and quantify the effect of geometry on the ejection efficiencies. We have assumed that the disk of the galaxy is described by a Miyamoto-Nagai potential, which is inversely proportional to $\left[R^{2}+\left(a+\sqrt{z^{2}+b^{2}}\right)^{2}\right]^{1 / 2}$, where $R, z$ are the cylindrical radial and vertical coordinates, respectively, and $a$ and $b$ are length scales. For $b / a \rightarrow 0$, the potential tends to the Kuzmin potential of a razor-thin disk, whereas for $b / a \rightarrow \infty$ it tends to the Plummer's spherical potential. We have taken 


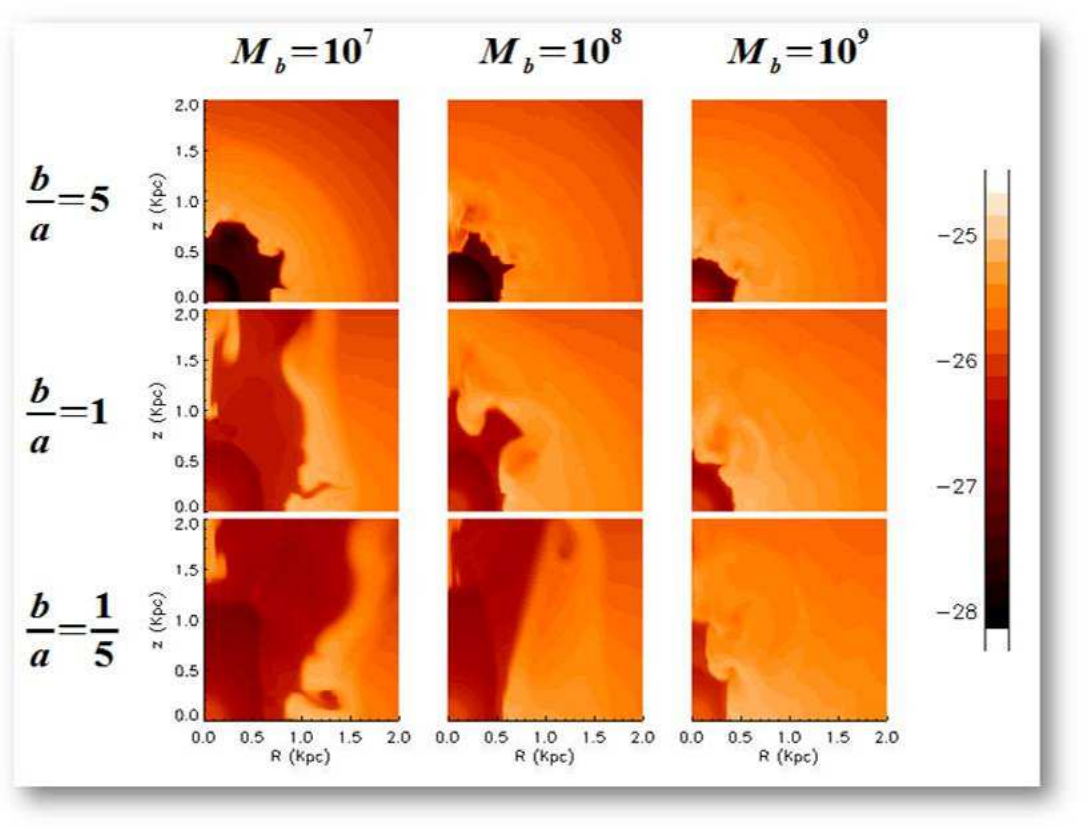

Figure 2: Gas distribution for the 9 models described in the text and depending on the degree of flattening (increasing from top row to bottom row) and on the total baryonic mass (increasing from left column to righ column). The (logarithmic) density scale is on the left-hand strip.

into account three possible values of the $b / a$ ratio: $b / a=0.2$ (thin disk), $b / a=1$ (thick disk) and $b / a=5$ (roundish galaxy). We have considered also three values of the total baryonic mass $\left(10^{7}\right.$, $10^{8}$ and $10^{9} \mathrm{M}_{\odot}$ ). The SF rate has been assumed constant (and equal to $2.5 \cdot 10^{-2} \mathrm{M}_{\odot} \mathrm{yr}^{-1}$ ). The evolution of the galaxy is followed for $500 \mathrm{Myr}$. Although we have explored a much wider parameter space [8], for the sake of illustration (and due to lack of space) here we show only the results related to these 9 basic models.

The gas distribution for these models after 200 Myr of evolution is shown in Fig. 2. It is evident already from this figure that, as outlined in many previous studies (see e.g. [4]), the development of large-scale outflows depends on the galactic mass. Since for the models with $10^{7} \mathrm{M}_{\odot}$ the potential well is shallower, the outflows are more powerful and a large fraction of gas and metals (more than 90\%) can be expelled from the galaxy. On the other hand, the behaviour of models with $10^{8}$ and $10^{9} \mathrm{M}_{\odot}$ strongly depends on the degree of flattening. All the models (including the ones developing large-scale outflows) are quite ineffective at removing gas from the galaxy (the transport of gas along the disk is very limited). However, outflows are clearly visible for thin disk (and even thick disk) models. Through these outflows, a large fraction of freshly produced metals (more than $80 \%$ for the flattened models with $10^{8} \mathrm{M}_{\odot}$ ) can be channelled out of the galaxy. To make the comparison clearer, let us take the models with $10^{9} \mathrm{M}_{\odot}$ and indicate with F, I, R the flat $(b / a=0.2)$, intermediate $(b / a=1)$ and roundish $(b / a=5)$ models, respectively. The amount of ejected oxygen at the end of the simulation for the three models are 64\% (F), 33\% (I), 16\% (R). Geometry can thus change the ejection efficiency of freshly produced metals by a factor of four. On the other hand, all these three models are able to eject only $\sim 10 \%$ of gas, with very little dependence on the $b / a$ ratio. Consequently, flat models will be four times less enriched in metals than roundish models 
from the ongoing SF episode.

\section{References}

[1] Chiappini, C., Hirschi, Meynet, G., et al. 2006, 449, L27

[2] Hensler, G., Theis, C., \& Gallagher, J.S. 2004, A\&A, 426, 25

[3] Koeppen, J., \& Hensler, G. 2005, A\&A, 434, 531

[4] MacLow, M.-M. \& Ferrara, A. 1999, ApJ, 513, 142

[5] Mühle, S., Klein, U.,Wilcots, E. M., \& Hüttermeister, S. 2005, AJ, 130, 524

[6] Recchi, S., \& Hensler, G. 2006, A\&A, 445, 875

[7] Recchi, S., \& Hensler, G. 2007, A\&A, 476, 841

[8] Recchi, S., \& Hensler, G. 2013, A\&A, in press

[9] Recchi, S., Hensler, G., \& Anelli, D. 2009, arXiv:0901.1976

[10] Schroyen, J., de Rijcke, S., Valcke, S., et al. 2011, MNRAS, 416, 601

[11] Silich, S., \& Tenorio-Tagle, G. 2001, ApJ, 552, 91

[12] Tolstoy, E., Hill, V., \& Tosi, M. 2009, ARA\&A, 47, 371

[13] Tremonti, C.A., Heckman, T.M., Kauffmann, G., et al. 2004, ApJ, 613, 898

[14] van Zee, L., Salzer, J.J., \& Haynes M.P. 1998, ApJ, 497, L1 